\title{
Caractérisation de l'aptitude fromagère des laits de brebis Lacaune à l'aide d'un Formagraph
}

\author{
A Delacroix-Buchet ${ }^{1}$, F Barillet ${ }^{2}$, G Lagriffoul ${ }^{3}$ \\ 1 Station de recherches laitières, INRA, 78352 Jouy-en-Josas cedex; \\ 2 Station d'amélioration génétique des animaux, INRA Auzeville, \\ BP 27, 31326 Castanet-Tolosan cedex; \\ ${ }^{3}$ Station d'amélioration génétique des animaux, CNBL Auzeville, \\ BP 27, 31326 Castanet-Tolosan cedex, France
}

(Reçu le 8 janvier 1993; accepté le 9 février 1994)

\begin{abstract}
Résumé - La présente étude visait à définir un protocole d'utilisation du Formagraph sur laits crus individuels non standardisés de brebis laitières. Des paramètres rhéologiques $(R, k 20, k 30$, aR) et physicochimiques $(\mathrm{pH}$, taux protéique et butyreux) ont été mesurés sur 146 laits individuels entiers de 20 brebis Lacaune suivies pendant toute leur lactation. L'influence respective du pH et du stade de lactation est estimée, en même temps que la répétabilité des caractères contrôlés au cours de la lactation, de même que les corrélations phénotypiques entre toutes les mesures. En l'absence de standardisation des laits, il est indispensable de mesurer simultanément le $\mathrm{pH}$ et les paramètres rhéologiques, dans la mesure où le $\mathrm{pH}$ est un facteur de variation majeur des résultats rhéologiques. Les corrélations phénotypiques entre paramètres rhéologiques et composition du lait sont cohérentes avec celles observées pour le lait de vache. En particulier, la fermeté des gels est corrélée positivement $(+0,59)$ au taux protéique. La variabilité individuelle des résultats et la répétabilité des mesures de paramètres rhéologiques, comparables à celles des taux protéiques et butyreux, est discutée du point de vue génétique.
\end{abstract}

lait individuel de brebis / coagulation à la présure / stade de lactation / corrélations phénotypiques

Summary - Characterization of cheese making properties of individual Lacaune ewe milk samples with a Formagraph. The present study aimed at defining a method using the Formagraph apparatus with non-standardized individual ewe milk samples. Rheological $(R, k 20, k 30, a R)$ and chemical ( $\mathrm{pH}$, protein and fat contents) parameters were determined on 146 individual whole milk samples from 20 Lacaune ewes sampled all along the lactation. Respective effects of $\mathrm{pH}$ and lactation stage were estimated simultaneously with the repeatability of different traits from ewes recorded all along their lactation, and the phenotypic correlations between all the measurements, using a mixed model. Without milk standardization, it is essential to measure $\mathrm{pH}$ and rheological parameters at the same time, because $\mathrm{pH}$ is one of the main factors of variation of rheological results. Phenotypic correlations between rheological parameters and milk composition were in agreement with those published in cow milk. In particular, curd firmness is positively correlated with protein content $(+0.59)$. Individual variability and repeatability of results of rheological parameters, comparable with those of the protein and fat contents, are discussed from a genetic point of view. 


\section{INTRODUCTION}

Le lait de brebis, essentiellement destiné à la production fromagère, se distingue du lait de vache par sa richesse en composants fromagers. En France, la sélection des brebis laitières, hormis la race Lacaune depuis 1985, porte uniquement sur la quantité de lait. Cette situation s'explique d'une part par le coût de mise en œuvre du contrôle qualitatif (taux butyreux: TB et taux protéique : TP) en ferme dans l'espèce ovine, d'autre part par la mise en place plus récente des schémas de sélection pour les autres races de brebis laitières. Pour la transformation fromagère, les effets de seuil sur les constituants des laits de brebis sont différents de ceux considérés habituellement en laits de vache et chèvre (seuil de TP). L'absence de standardisation des laits de brebis pour les fromages d'appellation d'origine contrôlée ainsi que la nécessité de respecter un pourcentage de gras sur sec minimum pour ces produits, imposent de tenir compte du rapport TB/TP (et non du TP seul), en plus bien sûr du taux de matière sèche utile $(T M S U=T B+T P)$ pour le rendement fromager.

Contrairement au lait de vache, le lait de brebis a été peu étudié. La revue bibliographique d'Assenat (1985) résume l'état des connaissances sur la composition et les propriétés du lait de brebis. Le lait de brebis contient globalement 2 fois plus de matière grasse que les laits de vache ou de chèvre et presque 2 fois plus de matière azotée totale. Sa teneur en lactose est légèrement inférieure à celle du lait de vache. Du fait de la saisonnalité de la production, le rapport et la somme des taux butyreux et azoté des laits de brebis évoluent fortement au cours de la période laitière : ils varient conjointement, plus faibles en début de saison et forts en fin de lactation. Mais comme pour les autres espèces laitières, les coefficients de variations phénotypiques des taux butyreux et protéique, calculés sur les fichiers de contrôle laitier, sont voisins respectivement de 10 et $5 \%$. Le lait de brebis est 2 fois plus minéralisé que le lait de vache, ce qui lui confère un fort pouvoir tampon et constitue un avantage pour sa conservation.

La connaissance des mesures de routine de la composition du lait (TB, TP) est nécessaire pour décrire rapidement les aptitudes fromagères du lait, mais insuffisante pour aborder de façon analytique les questions que se posent les fromagers (amélioration des rendements et de la qualité). Des études récentes permettent d'établir des relations entre les caractères physico-chimiques des laits de chèvre ou de vache et leur aptitude fromagère (Remeuf et al, 1989; 1991). Les données disponibles sur l'aptitude fromagère des laits de brebis sont très peu nombreuses. Par comparaison aux laits bovin ou caprin, le lait de brebis, en coagulation par la présure, a un temps de gélification court, le gel s'organise vite et devient très ferme (Storry et al, 1983). Cette fermeté est beaucoup plus importante que ne le laisserait supposer le rapport entre richesses fromagères des laits de vache et de brebis (Assenat, 1985). Ubertalle et al (1990) obtiennent des corrélations très significatives entre temps de gélification, vitesse de raffermissement et fermeté des gels. Le gel ovin s'égoutte moins rapidement que les gels bovin et caprin, ce qui s'expliquerait par sa grande concentration en matière grasse qui freine la synérèse (Storry et al, 1983).

Le présent travail s'inscrit dans le cadre d'un programme de recherches sur l'alimentation et la sélection de brebis laitières en vue de la valorisation fromagère du lait. Son objectif était de participer à la mise en place de tests permettant d'apprécier l'aptitude fromagère des laits. Dans l'article, nous nous intéressons au protocole d'utilisation du Formagraph, appareil de mesure rhéologique empirique couramment utilisé pour évaluer les paramètres rhéologiques des 
laits de vaches en coagulation présure. Le Formagraph a été appliqué avec succès notamment pour le contrôle de l'aptitude fromagère des laits destinés à la fabrication du Parmigiano Reggiano (Zannoni et Annibaldi, 1981 ; Zannoni et al, 1981). La variabilité des paramètres mesurés avec le Formagraph sur des laits individuels de brebis a été évaluée sur une vingtaine d'animaux de race Lacaune suivis pendant toute une lactation. La race Lacaune, exploitée dans le rayon de Roquefort, représente la première race de brebis traites en France. Dans un premier temps, nous analysons conjointement les facteurs de variation et la répétabilité de l'ensemble des paramètres rhéológiques mesurés avec le Formagraph sur les laits individuels. Puis, nous estimons et discutons des corrélations phénotypiques entre tous ces paramètres.

\section{MATÉRIEL ET MÉTHODES}

\section{Protocole expérimental}

Des échantillons individuels, qualitativement représentatifs de la traite du matin (automate de collecte), d'une vingtaine de brebis Lacaune du troupeau du domaine expérimental INRA de la Fage (Aveyron) ont été collectés pendant la campagne laitière 1989. Les prélèvements de laits ont été effectués sur le même groupe d'une vingtaine d'animaux, environ tous les $15 \mathrm{j}$, de février à juillet, de sorte qu'un total de 11 séries de prélèvements a été analysé (tableau I).

Chaque échantillon d'environ $100 \mathrm{ml}$ de lait individuel a été fractionné en $2 ; 30 \mathrm{ml}$ destinés à la détermination des taux butyreux et protéique, ont été additionnés de bichromate de potassium (concentration finale $0,06 \%$ ) et envoyés au Laboratoire interprofessionnel d'analyse du lait d'Aurillac (Cantal). Le reste, destiné aux mesures de l'aptitude à la coagulation par la présure, additionné de 2000 UI de pénicilline/l de lait (pour prévenir tout développement bactérien), a été expédié du domaine de La Fage, à la station INRA de recherches en technologie et analyses laitières de Poligny (Jura) (de février à fin avril) ou à la station INRA de recherches laitières de Jouyen-Josas (Yvelines) (de début mai à juillet), dans un emballage contenant de la glace fondante, par paquet Chronopost, 2-3 h après la traite du matin. Le temps entre l'expédition et la réception des échantillons n'a pas dépassé $24 \mathrm{~h}$ et $36 \mathrm{~h}$ entre l'expédition et l'acquisition des résultats Formagraph, à l'exception de la série du 24 avril (série 6), analysée le 26 dans la matinée.

\section{Méthodes}

\section{Analyses physico-chimiques}

Les taux butyreux (TB) et protéique (TP) ont été déterminés par une méthode physique indirecte infrarouge étalonnée par rapport aux méthodes de références pour le lait de brebis (Grappin, 1986).

Le $\mathrm{pH}$ des échantillons a été mesuré, à température ambiante (environ $20^{\circ} \mathrm{C}$ ) avec une électrode combinée, au départ de la Fage et à l'arrivée à Poligny ou à Jouy-en-Josas, afin de contrôler, en partie, la conservation des échantillons au cours du transport. C'est ainsi que l'on constate que l'addition de pénicilline a été suffisante pour limiter le développement bactérien au cours du transport puisqu'aucune acidification des échantillons n'a été notée.

\section{Analyses rhéologiques}

Les analyses rhéologiques ont été effectuées sur le même appareil Formagraph (Foss Electric SA France, Paris) à Poligny et à Jouy-en-Josas, en suivant le protocole établi à partir d'essais préliminaires réalisés au printemps 1988 . Toutes les mesures ont été effectuées en double, au pH initial du lait, sur les laits entiers, après $1 \mathrm{~h}$ à la température d'emprésurage, pour permettre un rééquilibrage des équilibres minéraux modifiés par le transport au froid. La température d'emprésurage des laits était fixée à $32^{\circ} \mathrm{C}$. Le coagulant était un extrait de présure liquide Granday (Beaune) contenant de la chymosine active à $520 \mathrm{mg} /$ et dilué le jour même des mesures au Formagraph dans un tampon pipérazine hexahydrate $(0,025 \mathrm{~mol} / \mathrm{l})$ de $\mathrm{pH} 5,3$. La dose de coagulant était pour $10 \mathrm{ml}$ de lait de $200 \mu \mathrm{l}$ d'une solution d'extrait de présure à $1,25 \%(v / v)$ en tampon pipérazine. 
Tableau I. Répartition des prélèvements de laits de 20 brebis (matrice d'incidence) considérés pour l'analyse statistique $(n=146)$.

Distribution of milk samples from 20 ewes (incidence matrix) used for statistical analysis ( $n=146$ ).

\begin{tabular}{llllllllllllll}
\hline Nb Brebis & 1 & 2 & 3 & 4 & 5 & 6 & 7 & 8 & 9 & 10 & 11 & $\begin{array}{c}\text { Série } \\
\text { detal }\end{array}$ \\
& $1 / 2^{*}$ & $15 / 2$ & $1 / 3$ & $15 / 3$ & $5 / 4$ & $24 / 4$ & $16 / 5$ & $30 / 5$ & $6 / 6$ & $21 / 6$ & $11 / 7$ prélèvements \\
complets \\
par brebis
\end{tabular}

\begin{tabular}{|c|c|c|c|c|c|c|c|c|c|c|c|c|c|}
\hline 1 & 82031 & i & & & i & & & & i & & a & a & 6 \\
\hline 2 & 82668 & & & & & & & & & a & $a$ & a & 8 \\
\hline 3 & 83073 & & & & & & & i & & & & i & 9 \\
\hline 4 & 83629 & & & & & & & & & & & & 11 \\
\hline 5 & 84029 & a & a & $a$ & a & & & & & i & & & 6 \\
\hline 6 & 84183 & & & & & & & & a & a & a & a & 7 \\
\hline 7 & 85094 & & a & & i & & i & i & & & & i & 6 \\
\hline 8 & 85177 & i & i & & & & i & i & i & i & i & i & 3 \\
\hline 9 & 85284 & & & & & i & i & $i$ & i & i & i & & 5 \\
\hline 10 & 86053 & & & & & a & & & & & & & 10 \\
\hline 11 & 86281 & a & a & a & a & & & i & i & i & & i & 3 \\
\hline 12 & 86379 & $a$ & i & & & & & & & $\mathrm{i}$ & & & 8 \\
\hline 13 & 87050 & & & & & & i & & & & $\mathrm{i}$ & $\mathrm{i}$ & 8 \\
\hline 14 & 87107 & & $\mathrm{i}$ & & & & & & & & & & 10 \\
\hline 15 & 87124 & & i & & & & & & & & $\mathrm{i}$ & $\mathrm{i}$ & 8 \\
\hline 16 & 87161 & a & a & a & a & & & & & $\mathrm{i}$ & & & 6 \\
\hline 17 & 87203 & & & & & & $\mathrm{i}$ & & & $\mathrm{i}$ & & $\mathrm{i}$ & 8 \\
\hline 18 & 87261 & & & & & & $\mathrm{i}$ & & & $\mathrm{i}$ & $i$ & $\mathrm{i}$ & 7 \\
\hline 19 & 87293 & & & & & & & & i & $\mathrm{i}$ & & & 9 \\
\hline 20 & 87680 & a & & & a & & & i & & & & & 8 \\
\hline \multicolumn{14}{|c|}{ Données: } \\
\hline & ésentes & 15 & 16 & 17 & 16 & 19 & 20 & 20 & 20 & 18 & 17 & 17 & \\
\hline & mplètes & 13 & 12 & 17 & 14 & 18 & 14 & 13 & 15 & 9 & 12 & 9 & 146 \\
\hline \multicolumn{14}{|c|}{ Stade moyen de } \\
\hline lacte & ation (j) & 62 & 76 & 90 & 104 & 125 & 145 & 165 & 180 & 186 & 200 & 221 & \\
\hline
\end{tabular}

* Date. a échantillon de lait absent. i Données incomplètes sur l'échantillon analysé.

"Date. a Milk sample missing. ${ }^{i}$ Incomplete data for the sample.

Le jour des analyses rhéologiques, un lait témoin reconstitué à $12 \%$ à partir d'une poudre de lait écrémé low heat (INRA de Poligny) était analysé en même temps que les laits expérimentaux pour tester la dérive éventuelle de l'appareil ou la perte de force de la présure (ie la stabilité des mesures dans le temps).

À partir des formagrammes, on a mesuré les paramètres suivants : le temps de gélification des laits (R, en min) évalué comme le temps entre l'emprésurage et le début du raffermissement (écartement entre les branches de $0,5 \mathrm{~mm}$ ), le temps de raffermissement des gels pour obtenir une fermeté standard (exprimée en $\mathrm{mm}$ ) de 20 ou $30 \mathrm{~mm}$ (respectivement k20 et k30) ainsi que la fermeté des gels à 2 fois le temps de gélification $(\mathrm{aR}$, en $\mathrm{mm})$. Le principe de fonctionnement du Formagraph est donné par Mc Mahon et 
al (1982). La figure 1 montre un enregistrement type obtenu avec le Formagraph pour les laits de brebis dans les conditions de mesures décrites.

\section{Analyses statistiques}

De février à juillet 1989 , les mesures avec le Formagraph ont été acquises pour un lot de 20 brebis, dont les laits ont été analysés pour au moins 7 des 11 séries d'envois de laits (soit les $2 / 3$ des séries) (tableau I). Au total, 228 laits ont été prélevés. Parmi ces 228 laits, 172 laits possédaient des données complètes d'analyse rhéologique et 146 laits possédaient des données complètes d'analyse rhéologique $(R, k 20, k 30$ et aR) et de production laitière (quantité de lait, TB et TP) pour la traite du matin correspondante. Les clauses d'exclusion pouvaient correspondre soit à des données de rhéologie manquantes ou partielles lorsque R était trop grand et que k20, k30 et aR n'étaient par conséquent pas mesurables, soit à l'absence d'échantillon de lait pour les analyses de composition du lait (tableau I). En résumé, avec les 20 brebis ayant fait l'objet d'un suivi régulier lors des 11 séries de prélèvements, on disposait de 146 mesures complètes, soit une moyenne de 7 mesures par brebis du $60^{\mathrm{e}}$ au $220^{\mathrm{e}}$ j de lactation (tableau I).

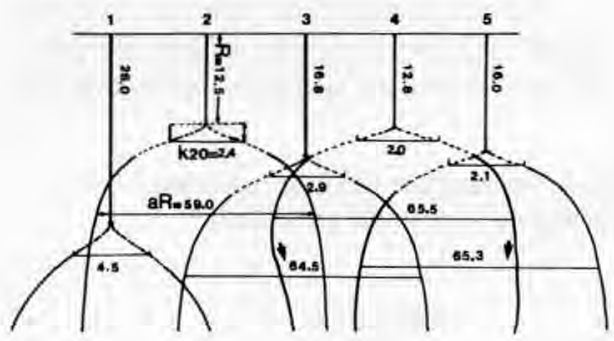

Fig 1. Formagrammes de laits individuels de brebis Lacaune (enregistrement type). R: temps de gélification (min); k20: temps de raffermissement du gel (min); aR: fermeté du gel à 2 fois le temps de gélification $(\mathrm{mm})$. Flèche : échantillon de lait $n^{\circ} 4$ : début de synérèse (rupture du gel).

Typical Formagrammes profile of individual Lacaune ewe's milk samples. R: rennet gelling time (min); k20: curd firming time (min); aR: curd firmness at twice the rennet gelling time (mm) Arrow: Sample milk number 4: beginning of syneresis (curd breakage).

\section{Répétabilité des doubles mesures rhéologiques}

Le calcul de la répétabilité entre doubles mesures réalisées avec le Formagraph sur les 172 laits individuels entiers a été effectué conformément à la norme AFNOR NF X-06041 (1970).

\section{Facteurs de variation, répétabilité des mesures successives et corrélations phénotypiques entre mesures}

Du point de vue génétique, pour un caractère mesuré plusieurs fois chez le même animal, on estime la répétabilité $\rho_{1}$ pour apprécier la ressemblance attendue entre les productions successives du même individu (Minvielle, 1990) : la répétabilité $\rho_{1}$ d'un caractère donné correspond à la proportion de la variance de ce caractère qui traduit des différences de nature permanente entre les animaux, incluant les effets génétiques et les effets d'environnement permanent propres à chaque individu. Par sa définition, la répétabilité (au sens génétique) constitue une limite supérieure de I'héritabilité.

Pour le calcul des coefficients de corrélation phénotypique et des coefficients de répétabilité entre mesures successives d'une même brebis, les 146 résultats de rhéologie et les performances laitières sont analysés selon le modèle mixte suivant :

$$
\begin{aligned}
Y_{i j k}= & \mu+b_{1}\left(p_{i j k}-p H\right) \\
& +S T A D E_{i}+B R E B I S_{j}+E_{i j k}
\end{aligned}
$$

oú $\mu$ est la moyenne des moindres carrés ; $b_{1}$ est la régression linéaire du $\mathrm{pH}$ individuel; STA$D E_{i}$, l'effet fixé du stade de lactation (11 niveaux) : BREBIS $_{j}$, l'effet aléatoire de la je brebis, de variance $\sigma_{B}^{2}$; $E_{i j k}$, l'effet aléatoire résiduel de variance $\sigma^{2} E$.

La régression $b_{1}$ n'est pas prise en compte lors de l'analyse du $\mathrm{pH}$ proprement dit.

Le coefficient de répétabilité pour un caractère 1 , entre mesures successives d'une même brebis à divers stades de lactation, est par définition égal à :

$$
\rho_{1}=\frac{\sigma_{B 1}^{2}}{\sigma_{B 1}^{2}+\sigma_{E 1}^{2}}
$$

Le coefficient de corrélation phénotypique entre 2 caractères 1 et 2 est égal à : 


$$
R p(1,2)=\frac{\sigma_{B 12}+\sigma_{E 12}}{\left(\sigma_{B 1}^{2}+\sigma_{E 1}^{2}\right)\left(\sigma_{B 2}^{2}+\sigma_{E 2}^{2}\right)}
$$

où $\sigma_{B 1}^{2}$ et $\sigma^{2} E 1$ sont les variances estimées de l'effet "brebis" et de l'erreur résiduelle du caractère 1, et $\sigma_{B 12}$ et $\sigma_{E 12}$ les covariances estimées de l'effet "brebis" et de l'erreur résiduelle entre les caractères 1 et 2 , selon la méthode du maximum de vraisemblance restreint. L'analyse des données a été réalisée à l'aide du logiciel SAS et de programmes spécifiques au département de génétique animale de l'INRA.

\section{RÉSULTATS}

\section{Répétabilité des doubles mesures rhéologiques}

L'écart type de répétabilité $\sigma_{\mathrm{r}}$, la répétabilité $r$ (exprimée par l'écart maximal obtenu entre doubles, $r=2,77 \sigma_{r}$ pour $P<0,05$ ) et le coefficient de variation en fonction de la moyenne $(\bar{X})$ des valeurs obtenues $\left(\mathrm{CV}_{\mathrm{r}}=\right.$ $\left.\left(\sigma_{r} / X\right) \times 100\right)$ sont présentés dans le tableau II. Puisque le temps de raffermissement des gels est très court, on a comparé la répétabilité des mesures de $k 20$ et $k 30$. On constate que le passage du k20 au k30 améliore peu la répétabilité des mesures ( $0,5 \%$ par rapport à la moyenne des valeurs mesurées), ce qui s'explique par l'organisation très rapide du gel. Par la suite, seuls les résultats concernant le $\mathrm{k} 20$ sont analysés. II apparaît donc que les mesures entre doubles de laits non écrémés sont répétables, en particulier le temps de gélification (R) et la fermeté des gels à 2 fois le temps de gélification $(\mathrm{aR})$ avec des coefficients de variation respectivement de 1,50 et $1,52 \%$.

\section{Statistiques descriptives}

Les paramètres de statistiques descriptives sont présentés au tableau III. Les brebis ont produit en moyenne $870 \mathrm{ml}$ de lait à la traite du matin, avec un TB moyen de $71,4 \mathrm{~g} / \mathrm{l}$ et un TP de $58,1 \mathrm{~g} / \mathrm{l}$, de sorte que le rapport TB/TP est égal à 1,22 .

Le pH de ces laits est en moyenne de 6,55 avec un coefficient de variation de $1,9 \%$, se traduisant par des extrêmes de 6,18 et 6,82 .

Si le temps moyen de gélification $(R)$ est égal à $12,7 \mathrm{~min}$, avec un coefficient de variation de $29,7 \%$, les extrêmes varient de 5 à

Tableau II. Répétabilité des paramètres rhéologiques du Formagraph (doubles mesures).

Repeatability of the rheological parameters of the Formagraph apparatus (duplicate).

\begin{tabular}{|c|c|c|c|c|}
\hline \multirow{2}{*}{$\begin{array}{l}\text { Variables } \\
(n=172)\end{array}$} & \multirow{2}{*}{$\begin{array}{l}\text { Moyenne } \\
(X)\end{array}$} & \multicolumn{3}{|c|}{ Répétabilité } \\
\hline & & $\begin{array}{c}\text { Écart type } \\
\left(\sigma_{r}\right)\end{array}$ & $\mathrm{r}=2,77 \sigma_{r}$ & $C_{V}=\left(\sigma_{r} \overline{(X}\right) \times 100$ \\
\hline$R(\min )$ & 12,9 & 0,19 & 0,54 & 1,50 \\
\hline $\mathrm{aR}(\mathrm{mm})$ & 56,7 & 0,86 & 2,38 & 1,52 \\
\hline k20 (min) & 2,4 & 0,16 & 0,44 & 6,55 \\
\hline k30 (min) & 3,6 & 0,22 & 0,60 & 6,08 \\
\hline
\end{tabular}


Tableau III. Statistiques descriptives des paramètres rhéologiques (Formagraph) et des mesures physico-chimiques des 146 prélèvements des 20 brebis.

Descriptive statistics of rheological parameters (Formagraph) and physico-chemical measurements of 146 milk samples from 20 ewes.

\begin{tabular}{|c|c|c|c|c|}
\hline & $\begin{array}{l}\text { Moyenne }(\bar{X}) \\
\text { (erreur type) }\end{array}$ & Minimum-maximum & $\begin{array}{l}\text { Écart type } \\
(\sigma)\end{array}$ & $C v=(\sigma / \bar{X}) \times 100$ \\
\hline $\mathrm{pH}$ & $\begin{array}{c}6,55 \\
(0,01)\end{array}$ & $6,18-6,82$ & 0,12 & 1,9 \\
\hline$R(\min )$ & $\begin{array}{l}12,7 \\
(0,3)\end{array}$ & $5,0-21,4$ & 3,8 & 29,7 \\
\hline $\mathrm{k} 20$ (min) & $\begin{array}{l}2,4 \\
(0,1)\end{array}$ & $1,3-4,9$ & 0,7 & 29,2 \\
\hline $\mathrm{R} / \mathrm{k} 20$ & $\begin{array}{c}5,40 \\
(0,08)\end{array}$ & $3,10-8,61$ & 0,98 & 18,1 \\
\hline $\mathrm{aR}(\mathrm{mm})$ & $\begin{array}{l}60,6 \\
(0,4)\end{array}$ & $43,0-71,0$ & 4,6 & 7,7 \\
\hline Lait matin (g) & $\begin{array}{l}869 \\
(33)\end{array}$ & $200-1750$ & 393 & 45,2 \\
\hline TB matin (g) & $\begin{array}{l}71,4 \\
(1,4)\end{array}$ & $23,5-139,8$ & 16,9 & 23,6 \\
\hline TP matin $(\mathrm{g})$ & $\begin{array}{l}58,1 \\
(0,7)\end{array}$ & $42,2-93,1$ & 8,5 & 14,5 \\
\hline $\mathrm{TB} / \mathrm{TP}$ matin & $\begin{array}{c}1,22 \\
(0,02)\end{array}$ & $0,51-1,65$ & 0,19 & 15,4 \\
\hline
\end{tabular}

plus de $30 \mathrm{~min}$. Parmi l'ensemble de 228 laits analysés, 2 étaient coagulés dès l'emprésurage ( $\mathrm{pH}$ acide de l'ordre de 6,2), et 12 n'étaient pas coagulés 30 min après l'emprésurage parmi lesquels 8 avaient un $\mathrm{pH}$ supérieur à 6,8.

La vitesse de raffermissement des laits de brebis emprésurés est très grande, ce qui se traduit par des valeurs de k20 très petites, de l'ordre de 2,4 min en moyenne.

La fermeté des gels à 2 fois le temps de gélification $(\mathrm{aR})$ est élevée. Pour une moyenne de $60,6 \mathrm{~mm}$, elle varie de 43 à 71 $\mathrm{mm}$, abstraction faite des laits présentant des temps de gélification trop grands pour que l'on puisse mesurer (aR) dans des délais raisonnables.

Le rapport R/k20, qui tient compte à la fois du temps de gélification et de la vitesse de raffermissement des gels est en moyenne égal à 5,40 , avec des variations du simple au triple $(3,10$ à 8,61$)$ ce qui illustre la variabilité des caractéristiques rhéologiques des laits individuels de brebis en cours de coagulation. 


\section{Effets des facteurs de variation liés au $\mathrm{pH}$ et au stade de lactation}

L'augmentation du TB à la traite du matin (+35 g/l) est plus marquée que celle du TP (+15 $\mathrm{g} / \mathrm{l})$ avec le stade de lactation (figs 2 et 3), de sorte que, de 60 à 220 j de lactation, le rapport TB/TP évolue en moyenne de 1,15 à 1,35 (fig 4). Pendant cette même période, la production laitière à la traite du matin régresse de 1,5 à 0,5 I (fig 5).

On constate par ailleurs une diminution marquée du $\mathrm{pH}$ des laits mesuré juste après la traite, vers le $140^{\mathrm{e}} \mathrm{j}$ de lactation, fin avrildébut mai (fig 6), ayant entraîné plus particulièrement une diminution du temps de gélification (R) et du k20, qui correspondent aux 2 paramètres rhéologiques les plus dépendants des variations de $\mathrm{pH}$. En fin de lactation, on observe une remontée des $\mathrm{pH}$.

Si l'on considère les données ajustées pour le $\mathrm{pH}$ conformément au modèle d'analyse (E1), on peut alors apprécier (indépendamment du $\mathrm{pH}$ ) les évolutions des paramètres rhéologiques avec le stade de lactation, qui reflètent indirectement les

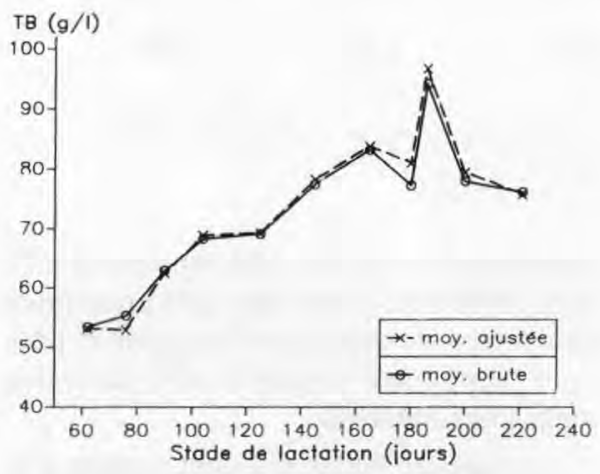

Fig 2. Évolution du taux butyreux (TB) moyen à la traite du matin des 146 prélèvements de lait (La Fage, 1989) (moyennes brutes et ajustées pour le $\mathrm{pH}$ ).

Evolution of the mean fat content at morning milking of 146 milk samples (La Fage, 1989) (uncorrected and $\mathrm{pH}$ adjusted means). variations de la composition chimique du lait (figs 2 et 3 pour le TB et le TP) : on constate ainsi, après ajustement, que le temps de gélification (R) augmente régulièrement du $80^{\circ}(R=9,4 \mathrm{~min})$ au $220^{\circ}$ jour de lactation ( $R=16,3 \mathrm{~min}$ ), soit environ $+1,4$ min tous les $30 \mathrm{j}$ de lactation (fig 7).

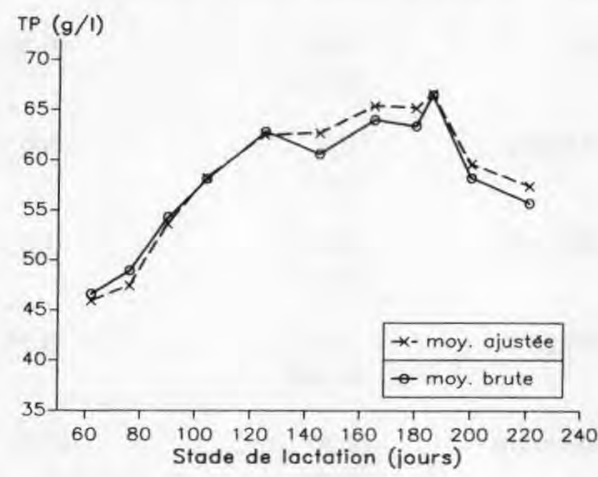

Fig 3. Évolution du taux protéique moyen à la traite du matin des 146 prélèvements de lait (La Fage,1989) (moyennes brutes et ajustées pour le $\mathrm{pH}$ ).

Evolution of the mean protein content at morning milking of 146 milk samples, La Fage 1989 (uncorrected and $\mathrm{pH}$ adjusted means).

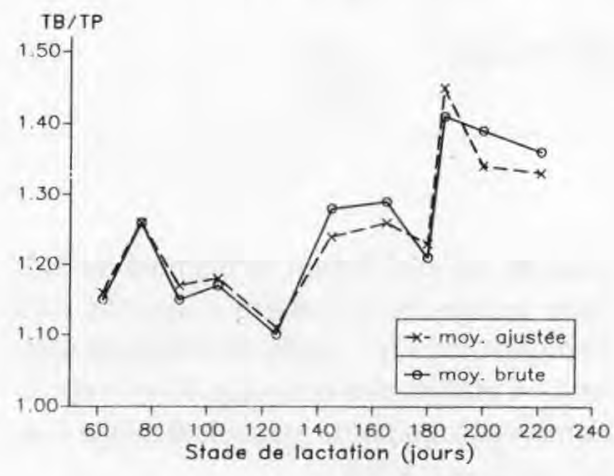

Fig 4. Évolution du rapport TB/TP moyen à la traite du matin des 146 prélèvements de lait (La Fage, 1989) (moyennes brutes et ajustées pour le $\mathrm{pH}$ ).

Evolution of the mean fat to protein ratio at morning milking of 146 milk samples, La Fage 1989 (uncorrected and $\mathrm{pH}$ adjusted means). 
De même, on constate une évolution régulière du k20 corrigé au $100^{\circ}(1,9 \mathrm{~min})$ au $200^{\mathrm{e}} \mathrm{j}$ de lactation (3,3 $\left.\mathrm{min}\right)$, après une phase initiale légèrement décroissante de 60 à $100 j$ de lactation. Si les autres paramètres rhéologiques $\mathrm{aR}$ et $\mathrm{R} / \mathrm{k} 20$ sont également affectés par le stade de lactation (figs 8 à 10), il est plus difficile de dégager une tendance régulière.

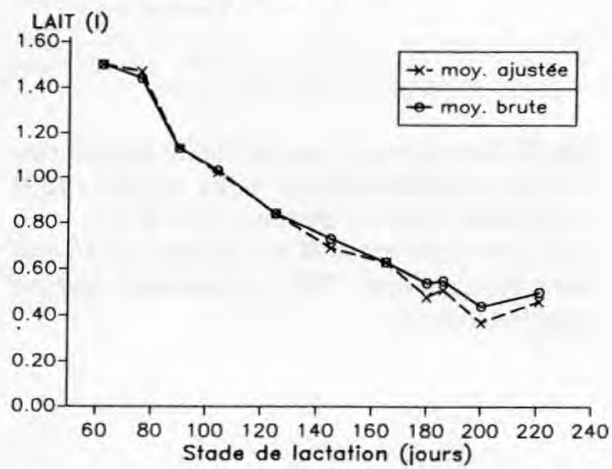

Fig 5. Évolution de la production laitière moyenne à la traite du matin correspondant aux 146 prélèvements de lait (La Fage, 1989) (moyennes brutes et ajustées pour le $\mathrm{pH}$ ).

Evolution of the mean yield of milk at morning milking corresponding to the 146 milk samples, La Fage 1989 (uncorrected and $\mathrm{pH}$ adjusted means).

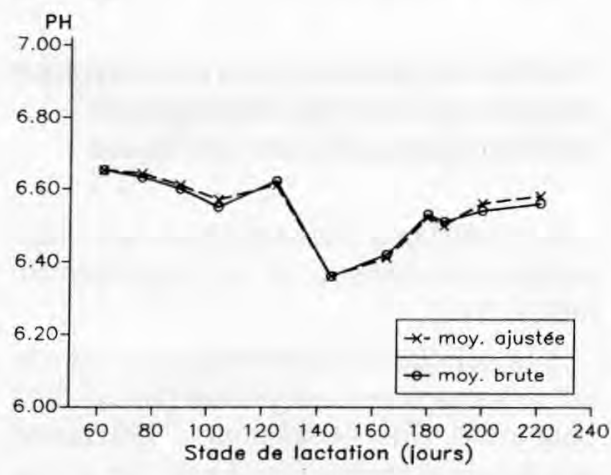

Fig 6. Évolution du pH moyen des 146 prélèvements de lait (La Fage, 1989) (moyennes brutes et ajustées pour le $\mathrm{pH}$ ). Evolution of the mean $\mathrm{pH}$ of 146 milk samples, La Fage 1989 (uncorrected and $\mathrm{pH}$ adjusted means).

\section{Répétabilité des mesures successives d'une même brebis}

En moyenne, chaque brebis fait l'objet de 7 doubles-mesures du $60^{\circ}$ au $220^{\circ}$ j de lactation (tableau I) : l'estimation de la repétabilité des mesures successives d'une même brebis, c'est-à-dire le coefficient de corré-

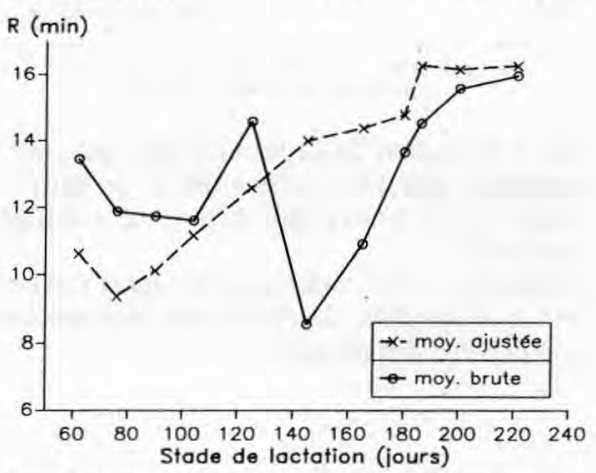

Fig 7. Évolution du temps de gélification (R) moyen des 146 prélèvements de lait (La Fage, 1989) (moyennes brutes et ajustées pour le $\mathrm{pH})$.

Evolution of the mean rennet gelling time $(R)$ of 146 milk samples, La Fage 1989 (uncorrected and $\mathrm{pH}$ adjusted means).

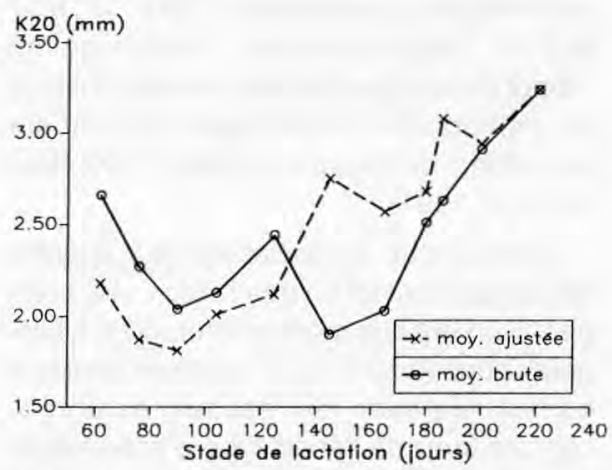

Fig 8. Evolution du temps de raffermissement (k20) moyen des 146 prélèvements de lait (La Fage, 1989) (moyennes brutes et ajustées pour le $\mathrm{pH}$ ).

Evolution of the mean curd firming time $(k 20)$ of 146 milk samples, La Fage 1989 (uncorrected and $\mathrm{pH}$ adjusted means). 


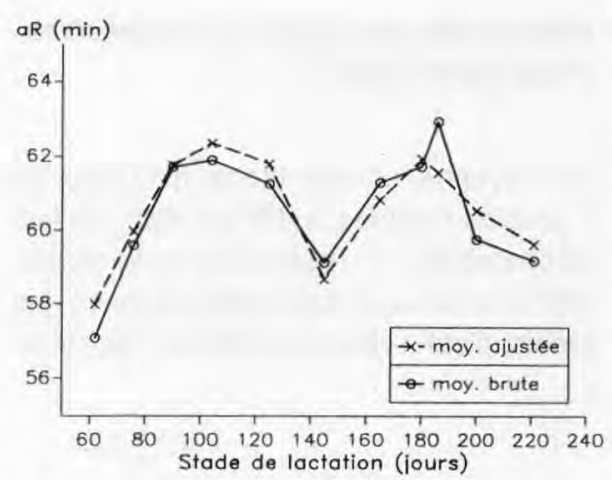

Fig 9. Évolution de la fermeté des gels (aR) moyenne des 146 prélèvements de lait (La Fage, 1989) (moyennes brutes et ajustées pour le $\mathrm{pH}$ ).

Evolution of the mean curd firmness (aR) of 146 milk samples, La Fage 1989 (uncorrected and $\mathrm{pH}$ adjusted means).

lation entre les mesures successives d'une même brebis, figure dans la diagonale du tableau IV pour les mesures physico-chimiques et les paramètres rhéologiques.

Si l'on s'intéresse d'abord aux mesures de production laitière, on obtient les valeurs estimées de la répétabilité du TB et TP, 0,57 et 0,67 respectivement, valeurs qui se situent dans la gamme des résultats obtenus sur fichiers de contrôle laitier portant sur des milliers de mesures (Barillet, 1990; Bouloc et al, 1991).

En revanche, la répétabilité de la quantité de lait apparaît ici un peu faible 0,44 , comparativement aux mêmes références bibliographiques $(0,50$ à 0,70$)$, mais elle demeure compatible avec les résultats habituels, compte tenu de l'écart type d'échantillonnage du résultat observé ici avec 146 mesures.

Le $\mathrm{pH}$ est la variable mesurée pour laquelle la répétabilité est la plus faible.

La répétabilité des mesures rhéologiques successives d'une même brebis est com-

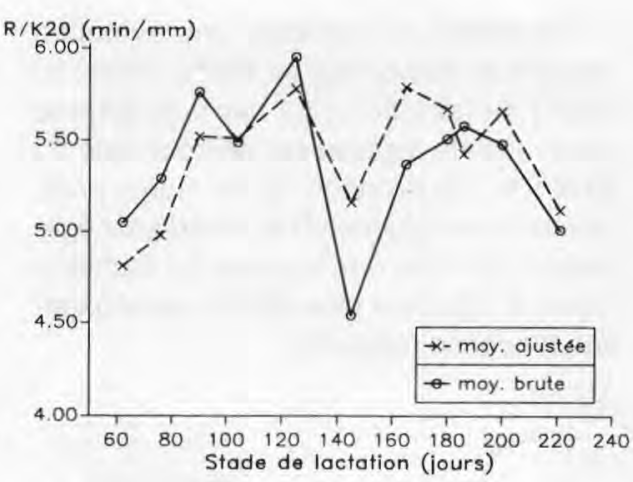

Fig 10. Évolution du rapport R/k20 moyen des 146 prélèvements de lait (La Fage, 1989) (moyennes brutes et ajustées pour le $\mathrm{pH}$ ).

Evolution of the mean $R$ to $k 20$ ratio of 146 milk samples, La Fage 1989 (uncorrected and $\mathrm{pH}$ adjusted means).

parable à celle du TB, voire du TP : ainsi la répétabilité des mesures successives d'une même brebis pour le temps de gélification $(\mathrm{R})$, la fermeté des gels (aR), le k20 et le rapport $R / k 20$, est estimée respectivement à $0,57,0,53,0,45$ et 0,48 .

\section{Coefficients de corrélation phénotypique entre les paramètres rhéologiques et les mesures physico-chimiques}

Les corrélations phénotypiques sont rapportées au-dessus de la diagonale du tableau IV.

Les corrélations phénotypiques entre le lait et les taux sont négatives $(R p=-0,43$ pour le $T B, R p=-0,45$ pour le TP), tandis que la liaison phénotypique entre les 2 taux est nettement positive $(R p=+0,72)$. Ces valeurs sont parfaitement en accord avec des résultats antérieurs acquis en race Lacaune sur des fichiers de plusieurs milliers de données (Barillet, 1985, 1989; Barillet et Boichard, 1987). 
Tableau IV. Coefficients de corrélations phénotypiques (au-dessus de la diagonale) et coefficients de répétabilité (sur la diagonale) entre les paramètres rhéologiques et les mesures physicochimiques ( 146 prélèvements de 20 brebis).

Phenotypic correlation coefficients (above the diagonal) and repeatability coefficients (on the diagonal) between rheological parameters and physico-chemical measurements (146 milk samples from 20 ewes).

\begin{tabular}{|c|c|c|c|c|c|c|c|c|c|}
\hline & $R$ & $a R$ & $k 20$ & $R / k 20$ & $p H$ & $T B$ & $T P$ & $T B / T P$ & Lait \\
\hline$R$ & $\begin{array}{l}+0,57 \\
(+0,10)\end{array}$ & $+0,28$ & $\stackrel{+0,60}{* * *}$ & $\stackrel{+0,41}{* *}$ & $\stackrel{+0,70}{* \star \star}$ & $+0,32$ & $+0,46$ & $+0,10$ & $-0,32$ \\
\hline aR & & $\begin{array}{l}+0,53 \\
(+0,10)\end{array}$ & $\begin{array}{l}-0,49 \\
* \star *\end{array}$ & $\stackrel{+0,85}{* * *}$ & $-0,09$ & $+0,27$ & $\stackrel{+0,59}{* \star \star}$ & $-0,07$ & $-0,20$ \\
\hline $\mathrm{k} 20$ & & & $\begin{array}{l}+0,45 \\
(+0,10)\end{array}$ & $\begin{array}{l}-0,46 \\
\star \star \star\end{array}$ & $+0,60$ & $+0,03$ & $-0,09$ & $+0,14$ & $-0,14$ \\
\hline $\mathrm{R} / \mathrm{k} 20$ & & & & $\begin{array}{l}+0,48 \\
(+0,10)\end{array}$ & $+0,24$ & $+0,32$ & ${ }_{*}^{+0,63}$ & $-0,06$ & $-0,21$ \\
\hline $\mathrm{pH}$ & & & & & $\begin{array}{l}+0,35 \\
(+0,08)\end{array}$ & $-0,12$ & $-0,05$ & $-0,10$ & $-0,10$ \\
\hline TB & & & & & & $\begin{array}{l}+0,57 \\
(+0,09)\end{array}$ & $\underset{\star \star \star \star}{+0,72}$ & $\underset{\star \star \star \star}{+0,62}$ & $-0,43$ \\
\hline TP & & & & & & & $\begin{array}{l}+0,67 \\
(+0,08)\end{array}$ & $+0,12$ & $\underset{\star \star}{-0,45}$ \\
\hline TB/TP & & & & & & & & $\begin{array}{l}+0,19 \\
(+0,09)\end{array}$ & $-0,21$ \\
\hline Lait & & & & & & & & & $\begin{array}{l}+0,44 \\
(+0,10)\end{array}$ \\
\hline
\end{tabular}

Modèle d'analyse: $(\mathrm{pH}+$ stade de lactation + brebis) pour toutes variables sauf $\mathrm{pH}$.

Modèle d'analyse: (stade de lactation + brebis) pour $\mathrm{pH}$.

*** $P<0,001 ;{ }^{* *} P<0,01 ;{ }^{*} P<0,05$.

Le temps de gélification $(R)$ est très dépendant du $\mathrm{pH}(\mathrm{R} p=+0,70)$, de même que le temps de raffermissement des gels (k20) $(R p=+0,60)$. Si $R$ et $k 20$ sont liés entre eux $(R p=+0,60)$, on constate cependant que $\mathrm{R}$ dépend de la composition chimique du lait $(R p=+0,46$ avec le TP, $R p=$ $+0,32$ avec le TB), à l'inverse de k20, qui est peu ou pas dépendant de cette dernière $(R p=-0,09$ avec TP, et $R p=+0,03$ avec TB).

La fermeté des gels à 2 fois le temps de gélification $(\mathrm{aR})$ dépend de $\mathrm{R} / \mathrm{k} 20$ ( $\mathrm{Rp}=$ $+0,85)$ et du taux protéique des laits $(R p=$ $+0,59)$. Le rapport $R / k 20$ est également lié au taux protéique des laits $(R p=+0,63)$. 


\section{DISCUSSION ET CONCLUSION}

Les résultats rhéologiques 1989 obtenus sur des laits de milieu de lactation confirment ceux obtenus en fin de lactation dans un essai préliminaire réalisé en 1988. Par ailleurs, les mesures de composition du lait de brebis à la traite du matin sont cohérentes avec les résultats antérieurs (Assenat, 1985 ; Barillet, 1985) : richesse du lait de brebis, exprimée en TB et TP, supérieure par rapport au lait de vache ou de chèvre, augmentation des taux butyreux et protéique et du rapport TB/TP des laits avec le stade de lactation, d'autant que le TP évolue beaucoup moins que le TB à partir du $120^{\circ}$ j de lactation.

Le $\mathrm{pH}$ des laits individuels de brebis est en moyenne inférieur à celui du lait de vache $(6,55$ vs 6,70$)$ (Alais, 1984). De plus, le $\mathrm{pH}$ constitue un facteur de variation majeur de la mesure du temps de gélification tout comme pour les laits de vaches : il serait donc en principe souhaitable de réaliser des mesures rhéologiques au $\mathrm{pH}$ initial $\left(\mathrm{pH}_{\mathrm{i}}\right)$ et à un $\mathrm{pH}$ standard $\left(\mathrm{pH}_{\mathrm{s}}\right)$ pour chaque lait. $\mathrm{Ce} \mathrm{pH}$ standard devrait être inférieur au $\mathrm{pH}_{\mathrm{s}}$ fixé à 6,60 pour les vaches dans le protocole INRA d'utilisation du Formagraph (document interne, 1989). Cependant, comme on a observé une large plage de variation des $\mathrm{pH}_{\mathrm{i}}$ des laits individuels de brebis, l'ajustement du $\mathrm{pH}$ de certains laits à une valeur $\mathrm{pH}_{\mathrm{s}}$ très inférieure à leur $\mathrm{pH}_{\mathrm{i}}$ risquerait de biaiser de façon non négligeable leur comportement au cours de la coagulation, et par suite leur réponse observée avec le Formagraph, si les quantités d'acide lactique à apporter étaient relativement grandes. Ces quantités d'acide seraient d'autant plus élevées que les laits de brebis possèdent un fort pouvoir tampon, par leur richesse en matières minérales. En pratique, il apparaît donc plus réaliste de travailler a $\mathrm{pH}_{\mathrm{i}}$, et de considérer obligatoirement cette covariable dans toute analyse de paramètres rhéologiques. On peut supposer aussi que les variations importantes de $\mathrm{pH}$ observées en avril-mai, puis en juillet, pourraient être mises en relation, respectivement avec la modification de régime alimentaire au printemps, et avec le stade de lactation avancé des brebis en fin de campagne.

Le temps de gélification est très variable et certains laits coagulent mal ou pas du tout (laits spécifiques à quelques brebis, exemples : 85177 et 85284 , et laits de fin de lactation; tableau I). Cette constatation ne permet pas d'envisager d'augmenter la précision des mesures de k20 par une augmentation du temps de gélification (température d'emprésurage de $30^{\circ} \mathrm{C}$ au lieu de $32^{\circ} \mathrm{C}$, diminution de la dose de coagulant) ce qui augmenterait globalement les temps de mesures et surtout le nombre d'échantillons qui ne coagulent pas dans les conditions du protocole.

Les paramètres $\mathrm{k} 20$ et aR n'ont quelquefois pas pu être mesurés du fait de la mauvaise aptitude à la coagulation des laits ou au contraire de l'organisation trop rapide des gels puis de leur rupture (exemple du lait 4, fig 1).

Le rapport R/k20 est en moyenne plus de 2 fois supérieur à celui des laits de vache (Vertes, 1989).

Les corrélations phénotypiques entre paramètres rhéologiques sont cohérentes avec les corrélations mentionnées dans la bibliographie pour le lait de vache ou par Ubertalle et al (1990) pour le lait de brebis de race Delle Langhe. En particulier, les corrélations phénotypiques entre le TB et les paramètres rhéologiques mesurés avec le Formagraph qui sont comprises entre 0,03 et 0,3 sont nettement plus faibles que les corrélations phénotypiques entre le TP des laits et certaines mesures telles la fermeté des gels $(R p=0,59)$ ou le rapport $R / k 20(R p=0,63)$, ce qui rejoint les observations de Storry et al (1983).

Par ailleurs, les paramètres rhéologiques présentent des coefficients de répétabilité 
comparables ou légèrement inférieurs à ceux des mesures de composition du lait : de 0,57 pour $(R)$ à 0,45 pour $(k 20)$, comparativement à 0,57 et 0,67 pour (TB) et (TP). Si les répétabilités de ces 2 groupes de caractères sont comparables, en revanche les corrélations phénotypiques entre composition du lait (TB,TP) et l'ensemble des paramètres rhéologiques ne sont pas assez élevées pour envisager une sélection indirecte efficace des paramètres rhéologiques, à partir d'une sélection directe sur la composition du lait. Sous l'hypothèse que ces mesures rhéologiques reflètent mieux les aptitudes fromagères du lait que sa composition chimique (TB, TP), on pourrait alors s'interroger sur la définition de critères rhéologiques mesurables dans le cadre du contrôle laitier. Pour envisager une telle sélection directe sur des critères rhéologiques, il faudrait cependant être en mesure de pratiquer ces tests rhéologiques sur des dizaines de milliers d'animaux à divers stades de lactation (exemple : 150000 brebis Lacaune en contrôle laitier officiel, à échantillonner 2 à 4 fois par lactation), avec mesure conjointe du $\mathrm{pH}$ individuel. Un tel scénario apparaît comme irréaliste, avec un appareil tel que le Formagraph. II supposerait en tout état de cause d'une part d'automatiser ces tests rhéologiques, d'autre part de les étalonner vis-à-vis des fabrications fromagères concernées, avant d'envisager la prise en compte de tels critères rhéologiques dans le cadre du contrôle laitier ovin. On pourrait aussi imaginer un deuxième scénario, où la sélection laitière classique sur le lait et sa composition (TB, TP) est maintenue, avec des vérifications régulières pour constater s'il y a évolution ou pas de la réponse rhéologique de laits de brebis élites du noyau de sélection (mères des béliers utilisés en insémination artificielle). Cette variante supposerait probablement aussi, outre la standardisation des tests, une automatisation des mesures autorisant la réalisation de centaines à quelques milliers de mesures annuelles. En pratique, il serait donc certainement plus réaliste d'envisager un test simple et peu coûteux, condition indispensable pour son application à grande échelle par tous les acteurs de la filière (éleveurs, fromagers...), fondé sur la seule mesure du temps de gélification.

En tout état de cause, conformément à notre objectif initial, nous disposons donc, à l'issue de ce travail, d'un protocole d'utilisation du Formagraph avec des laits entiers individuels de brebis, c'est-à-dire d'un outil analytique permettant d'étudier les aptitudes fromagères du lait dans le cadre de recherches pluridisciplinaires.

\section{REMERCIEMENTS}

Les auteurs remercient MR Aurel (domaine expérimental INRA de La Fage, Aveyron) qui a réalisé l'ensemble des prélèvements et des envois de laits nécessaires à l'étude financée dans le cadre d'une AIP "qualité du lait" de l'INRA.

\section{RÉFÉRENCES}

AFNOR (1970) Fidélité d'une méthode d'essai. Guide pour les essais interlaboratoires. Norme NF X06-041

Alais C (1984) Science du lait, $4^{e}$ ed. Sepaic, Paris

Assenat L (1985) Le lait de brebis: composition et propriétés. In: Lait et produits laitiers I. (Luquet FM, ed). Lavoisier-APRIA, Paris, 281-319

Barillet $F$ (1985) Amélioration génétique de la composition du lait des brebis: l'exemple de la race Lacaune. Thèse INA Paris Grignon

Barillet $F(1989)$ Expression de la production laitière à la traite des brebis Lacaune en système allaitement $x$ traite mécanique. Proc 4 th Int Symp machine milking of small ruminants, Tel-Aviv, Israël, 13-19 september, 463-495

Barillet F (1990) Rapport du groupe du CICPLB sur le contrôle laitier des brebis. La simplification du contrōle laitier officiel de type $A$. EAAP Publ 50, 119-125 
Barillet F, Boichard D (1987) Studies on dairy production of milking ewes. I. Estimates of genetic parameters for total milk composition and yield. Génét Sél Évol 19, 459-474

Bouloc N, Barillet F, Boichard D, Sigwald JP, Bridoux G (1991) Étude des possibilités d'allègement du contrôle laitier officiel chez les caprins. Ann Zootech 40, 125-139

Grappin R (1986) Application of indirect instrumental methods to the measurement of fat and protein contents of goat and ewe milk. Bull Fed Int Lait 202, 76-78

Mc Mahon DJ, Brown RJ (1982) Evaluation of Formagraph for comparing rennet solutions. J Dairy Sci 65, 1639-1642

Minvielle F (1990) Principes d'amélioration génétique des animaux domestiques. INRA, Paris et PUL, Québec, 1990

Remeuf F, Cossin V, Dervin C, Lenoir J, Tomassone R (1991) Relations entre les caractères physico-chimiques des laits et leur aptitude fromagère. Lait 71, 397-421
Remeuf F, Lenoir J, Duby C (1989) Étude des relations entre les caractéristiques physicochimiques des laits de chèvre et leur aptitude à la coagulation par la présure. Lait 69, 499-518

Storry JE, Grandison AS, Millard D, Owen AJ, Ford GD (1983) Chemical composition and coagulating properties of renneted milks from different breeds and species of ruminant. J Dairy Sci 50, 215-229

Ubertalle A, Ambrosoli R, Errante J (1990) Tippizzazione lattodinamographica del latte ovino. Sci Tec Latt-Casearia 41, 53-63

Vertes C (1989) Étude de l'influence de quelques facteurs zootechniques sur la composition physico-chimique et l'aptitude fromagère du lait de vache. Thèse de l' Université Rennes I

Zannoni M, Annibaldi S (1981) Standardization of the renneting ability of milk by Formagraph. I. Sci Tec Latt-Casearia 32, 79-94

Zannoni M, Mongardi M, Annibaldi S (1981) Standardization of the renneting ability of milk by Formagraph. II. Data processing. Sci Tec LattCasearia 32, 153-164 